\title{
Detection of Truth Discovery in Big Data Social Media Sensing Applications
}

\author{
A Hemadri Naidu, J Naga Muneiah
}

\begin{abstract}
With the rapid growth of online social media and ubiquitous Internet connectivity, social sensing has emerged as a new crowd sourcing application paradigm of collecting observations (often called claims) about the physical environment from humans or devices on their behalf. A fundamental problem in social sensing applications lies in effectively ascertaining the correctness of claims and the reliability of data sources without knowing either of them a priori, which is referred to as truth discovery. While significant progress has been made to solve the truth discovery problem, some important challenges have not been well addressed yet. First, existing truth discovery solutions did not fully solve the dynamic truth discovery problem where the ground truth of claims changes over time. Second, many current solutions are not scalable to large-scale social sensing events because of the centralized nature of their truth discovery algorithms. Third, the heterogeneity and unpredictability of the social sensing data traffic pose additional challenges to the resource allocation and system responsiveness. In this paper, we develop a Scalable and Robust Truth Discovery (SRTD) scheme to address the above three challenges. In particular, the SRTD scheme jointly quantifies both the reliability of sources and the credibility of claims using a principled approach. The evaluation results on three real-world data traces (i.e., Boston Bombing, Paris Shooting and College Football) show that the SSTD scheme is scalable and outperforms the state-of-the- art truth discovery methods in terms of both effectiveness and efficiency.
\end{abstract}

Keywords: Big Data, SRTD, Data Sparsity, Robust, Social Media Sensing

\section{INTRODUCTION}

T his paper presents a scalable streaming truth discovery scheme for social sensing applications. Social sensing has emerged as a new paradigm of crowdsourcing applications where humans are used as ubiquitous, versatile and inexpen- sive sensors to report their observations (often called claims) about the physical world [35]. This paradigm is motivated by the proliferation of portable data collection devices (e.g., smartphones), the wide adaptation of online social media

Revised Manuscript Received on December 30, 2019.

* Correspondence Author

Mr.A Hemadri Naidu*, M.Tech Student, Dept. of CSE, Chadalawada Ramanamma Engineering College, Tirupati, India. hemadrinaidu3@gmail.com

Prof. J Naga Muneiah, Head Department, of CSE, Chadalawada Ramanamma Engineering College, Tirupati, India. nagamuni513@gmail.com

(C) The Authors. Published by Blue Eyes Intelligence Engineering and Sciences Publication (BEIESP). This is an open access article under the CC BY-NC-ND license (http://creativecommons.org/licenses/by-nc-nd/4.0/) (e.g., Twitter, Facebook) and the ubiquitous Internet connectivity (e.g.,WiFi, 4G/5G). Examples of social sensing include ob- taining real-time situation awareness in disaster and emergency response scenarios [34], intelligent transportation system ap- plications using location based social network services [1], geotagging and urban sensing applications using inputs from common citizens [40]. A critical challenge in social sensing is referred to as truth discovery where the goal is to identify the reliability of the sources and the truthfulness of claims they make without the prior knowledge on either of them.

Consider a campus attack scenario (e.g., OSU attack in Nov. 2016) as an example. A significant amount of reports about the current situation of the attack (e.g., the number of casualties, the escape path of suspects and safety alerts) are available from the social sensors (e.g., news reporters and common citizens on social media). However, those social sensors may not always generate reliable claims and some of their claims may even contradict with each other. Table I shows some example tweets collected in the OSU attack. We observe the first two tweets report that there was a shooting happening at OSU campus while the third one report it was false news. In general, it is very challenging to identify the truthfulness of the claims without knowing the reliability of the individual sources who make them a priori. Additionally, sources could also intentionally or unintentionally propagate the misinformation through their social networks [38]. All these complexities make the truth discovery in social sensing a non-trivial task to accomplish.

Table-I: Example Tweets on Contradicting Claims in OSU Campus Attack, November, 2016.

\begin{tabular}{|c|c|}
\hline Tweet & Timestamp \\
\hline $\begin{array}{c}\text { OSU POSSIBLE SHOOTING: I am on campus } \\
\text { near @OSUengineering TONS of police. }\end{array}$ & 7:23 AM \\
\hline There was a shooting at Ohio state please pray & 28 Nov 2016, \\
for people's safety \#osu & $7: 47$ AM EST \\
\hline $\begin{array}{c}\text { Liberals putting out fake claims about the ter- } \\
\text { rorist attack. 1st not a shooting, 2nd not an }\end{array}$ & $\begin{array}{c}\text { Nov 2016, } \\
\text { 11:37 AM EST } \\
\text { American, 3rd not nazi but Islamic \#osushooting }\end{array}$ \\
\hline
\end{tabular}

A rich set of solutions have been proposed in network sensing, data mining, machine learning communities to solve the truth discovery problem [7], [14], [17], [25], [37], [39], [41]. However, several significant challenges have not been well addressed by the state-of-the-art solutions. First, existing solutions did not fully solve the dynamic truth discovery problem where the ground truth of claims changes over time. 
There are two critical tasks in addressing the dynamic truth challenge.

The first is to capture the transition of truth in a timely manner and the second one is to be robust against noisy data that may lead to the incorrect detection of the truth transition. Only a small number of schemes been proposed to solve the dynamic truth discovery problem. For example, Pal et. al considered the evolving information of objects and esti- mated the truth of variables in current time interval based on sources' historical claims [24]. Li et al. proposed a Maximum A Posterior based real-time algorithm to solve the dynamic truth discovery problem [9]. However, these approaches could be unresponsive when the amount of social sensing data is large or the amount of resources on the deployed system is limited. Moreover, their solutions are not robust in noisy and sparse social sensing scenarios since their truth discovery accuracy is sensitive to the quality and quantity of thesensing data.

Second, existing truth discovery solutions did not fully explore the scalability aspect of the truth discovery problem. Social sensing applications often generate large amounts of data during some important events (e.g., disasters, sports, unrests) [27]. For example, 3.8 million people have generated a total of 16.9 million tweets with tweet per minute peaked at a rate of over 152,000 in Super Bowl 2016 [22]. However, current centralized truth discovery solutions are incapable of handling such large volume of social sensing data due to the resource limitation on a single computing device. A limited number of distributed solutions have been developed to address the scalability issue of the truth discovery problem. Both Ouyang et al. [23] proposed a distributed solution based on Hadoop system, but there are several non-trivial drawbacks. First, Hadoop is a heavy-weight solution in the sense that it requires a long start up time. Second, Hadoop is designed as a batch processing system that is most suitable for data of very large volume (e.g., Petabytes of data) and may not be the best solution for the size of datasets collected in many social sensing events (e.g., GB to TB). Third, Hadoop assumes homogeneity of the underlying of the computational resources we have in real distributed systems.

The third challenge lies in the heterogeneity and unpredictability of the streaming data traffic. First, different topics partitions can be processed in a synchronized manner [23]. However, such strong homogeneity assumption on the data streams barely holds in real world social sensing applications. In this paper, we develop a Scalable Streaming Truth Discovery (SSTD) scheme to address the above challenges. To address the dynamic truth discovery challenge, we develop a Hidden Markov Model based solution to dynamically estimate the true value of claims based on the observations reported by social sensors. To address the scalability challenge, we developed a light-weight distributed framework that is both scalable and efficient to solve the truth discovery problem using Work Queue and HTCondor system. To address the data heterogeneity challenge, we computing nodes [29], which ignores the heterogeneity

integrated the Scalable and Robust Truth Discovery (SRTD) scheme with an optimal workload allocation mechanism using feedback control (i.e., Proportional Integral Derivative (PID) controller) to dynamically allocate the resources (e.g., cores, memories) to the truth discovery tasks. We evaluated the Scalable and Robust Truth Discovery (SRTD) scheme in comparison with the state-of-the-art truth discovery baselines using three real-world social sensing data traces (i.e., Boston Bombing, Paris Shooting and College Football) collected from Twitter. The evaluation results show that our Scalable and Robust Truth Discovery (SRTD) scheme significantly outperforms the compared baselines in terms of truth discovery accuracy and computational efficiency.

In summary, the contributions of this paper are as follows:

- This paper addresses three fundamental challenges in truth discovery problem in social sensing: dynamic truth, scalability and heterogeneity of streaming data.

We develop the Scalable and Robust Truth Discovery (SRTD) scheme that incorporates the Hidden Markov Model (HMM) to effectively address the dynamic truth discovery challenge.

-We develop a light-weight distributed framework based on Work Queue and HTCondor system to address the scalability challenge.

We integrate the Scalable and Robust Truth Discovery (SRTD) scheme with an optimal workload allocation mechanism to address the heterogeneity of the streaming social sensing data.

-We evaluate the performance of the Scalable and Robust Truth Discovery (SRTD) scheme and compare it with the state-of-the-art truth discovery solutions through real-world case studies. The evaluation results demonstrate the effectiveness and significant per- formance gains achieved by our scheme.

\section{PROBLEM FORMULATION}

In this section, we formulate our robust truth discovery problem in big data social media sensing. In particular, consider a social media sensing application where a group of $M$ sources $S=\left(S_{1}, S_{2}, \ldots, S_{M}\right)$ reports a set of $N$ claims, namely, $C=\left(C_{1}, C_{2}, \ldots, C_{N}\right)$. Let $S_{i}$ denote the $i$ th source and $C_{j}$ denote the $j$ th claim. We define $R P^{t}$ to be the report made by source $S_{\boldsymbol{i}}$ on claim $C_{\boldsymbol{j}}$ at time $t$. Take, Twitter as an example; a source refers to a user ac-count and a claim is a statement of an event, object, or topic that is derived from the source's tweet. For example, a tweet "Not much of the comment about the Dallas shooting has focused on the fact the sniper was a veteran." is associated with a claim "Dallas shooting sniper was a veteran". The tweet itself is considered as the report. We observe that the social media sensing data is often sparse (i.e., the majority of sources only contribute to a limited number of claims in an event). We further define $C_{j}=T$ and $C_{j}=F$ to represent thata claimistrueorfalse, respectively.Eachclaimisalso associated with a ground truth label $x_{j}^{*}$ such that $x_{j}=1$ when $C_{j}$ is true and $x_{j}=0$ otherwise. 
The goal of the truth discovery task is to jointly estimate the truthfulness of each claim and the reliability of each source, which is defined as follows:

DEFINITION 1. Claim Truthfulness $D_{j}$ for claim $C_{j}$ : The likelihood of a claim to be true. The higher $D_{j}$ is, the more likely the claim $C_{\boldsymbol{j}}$ is true.

Formally we define $D_{j}$ to estimate:

$\operatorname{Pr}\left(C_{\boldsymbol{j}}=T\right)$

DEFINITION 2. Source Reliability $R_{i}$ for source $S_{i}$ : Ascore represents how trustworthy a source is. Thehigher $R_{i}$ is, the more likely the source $S_{i}$ will provide credible and trustworthy information. Formally we define $R_{i}$ to estimate:

$$
\operatorname{Pr}\left(C_{j}=T \mid S C_{i, j}=T\right)
$$

where $S C_{i, j}=T$ denotes that source $S_{i}$ reports claim $C_{j}$ to be true.

Since sources are often unvetted in social media sensing applications and may not always report truthful claims, we need to explicitly model the reliability of data sources in our problem formulation. However, it is challenging to accurately estimate the reliability of sources when the social media sensing data is sparse [34]. Fortunately, the reports themselves often contain extra evidence and information to infer the truthfulness of a claim. In the Twitter example, the text, pictures, URL links, and geotags contained in the tweet can all be considered as extra evidence of the report. To leverage such evidence in our model, we define a credibility score for each report to represent how much the report contributes to the truthfulness of a claim.

We first define the following terms related to the credibility score of a report made by source $S_{i}$ on claim $C_{j}$ at time $k$.

DEFINITION 3. Attitude Score ( $\rho \mathrm{k})$ : Whether a source believes the claim is true, false or does not provide any report. We use $1,-1$ and 0 to represent these attitudes respectively.

DEFINITION 4: Hidden States of Truth: the true value for the claim at a given time instant that is not directly observable.

DEFINITION 5. Independent Score: ( $\eta \mathrm{k}$ ): A score in the range of $(0,1)$ that measures whether the report Ri,u is made independently or copied from other sources. A higher score is assigned to a report that is more likely to be made independently.

\section{PROPOSED ALGORITHMS}

This Paper describes the three challenges nothing but the Misinformation, data sparsity and trustworthiness using the SRTD(Scalable and robust trust discovery scheme). Certain observations are made which are relevant to our model as follows

$\checkmark$ Observation 1: Sources often spread false information from others without independent verification by simply copying or forwarding information (e.g., retweets on Twitter).

$\checkmark$ Observation 2: False claims have intensive debates on those claims and often controversial and sources tend to disagree with each other.

$\checkmark$ Observation 3: If a source debunk sits previous claim, it's very likely the previous claim is false because people are generally prone to be self-consistent.

More specifically, the contribution score of source $\mathrm{Si}$ on claim $\mathrm{Cj}$ is denoted as CSij and it is formally calculated as: $C S i j=\operatorname{sgn}(\operatorname{SLSK} i, j) K$ $\mathrm{X}$ k=1RK+1-k i |SLSki,j|

The following diagram explains about the SRTD architecture

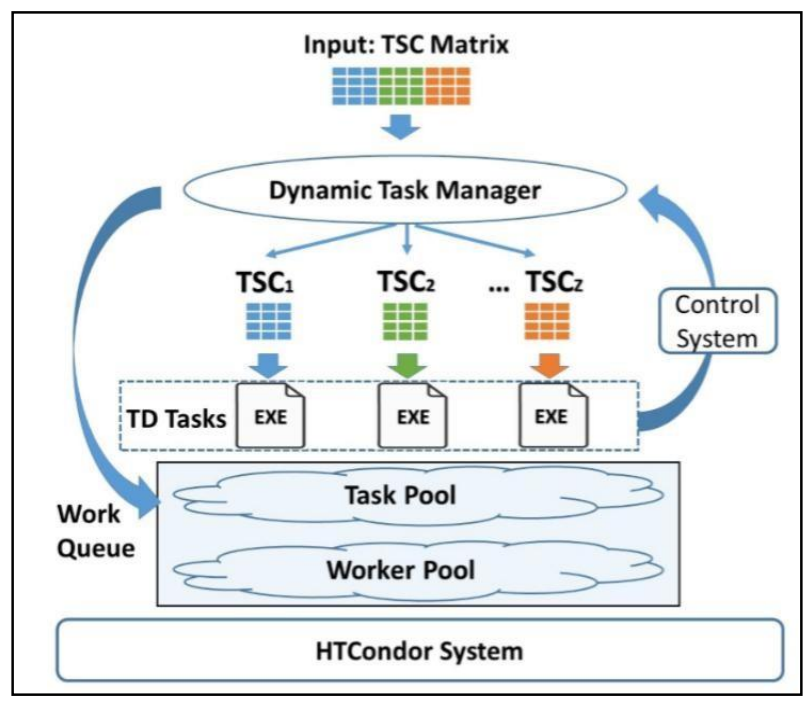

\section{A. Architecture of SRTD}

Usually from the above diagram Dynamic Task Manager which implemented as a master work Queue process that initializes a work pool, dynamically spawns new task into the task pool and it considered as a key component. DTM divides the TSC matrix into sub matrices then it spawns set4 of tasks to process all submatrices on the HTCondor system. SRTD is always integrated with the feedback control system to monitor the current execution speed of each Truth Discovery task and also to estimate its expected finish time. DTM was informed by the feedback control system of control signals based on the performance of the system and it dynamically adjust the task priority and resource allocation to optimize the overall system performance.

\section{B. SRTD iAlgorithm}

1) Algorithm 1 Scalable Robust Truth Discovery (SRTD)

Input: TSC matrix

Output: claim truthfulness ^ $\mathrm{x} * \mathrm{j}, \forall 1 \leq \mathrm{j} \leq \mathrm{N}$

Initialize $\mathrm{Ri}=0.5, \forall \mathrm{i} \leq \mathrm{M}$; set the values of credibility scores; initialize max iteration $=100$

Split Original TSC matrix into Z submatrices, let S(z) denote the number of sources in the $z$-th submatrix while $\{\mathrm{Dj}\}$ do not converge or reach max iteration do

for all $\mathrm{z}, 1 \leq \mathrm{z} \leq \mathrm{Z}$ do for all $\mathrm{i}, 1 \leq \mathrm{i} \leq \mathrm{S}$ (z) do for all $\mathrm{j}, 1 \leq \mathrm{j} \leq \mathrm{N}$ do

\section{if TSCij exists then}

compute CSij based on Equation (6)

end if

end for

end for

for all $\mathrm{i}, 1 \leq \mathrm{i} \leq \mathrm{S}(\mathrm{z})$ do

estimate Ri based on Equation

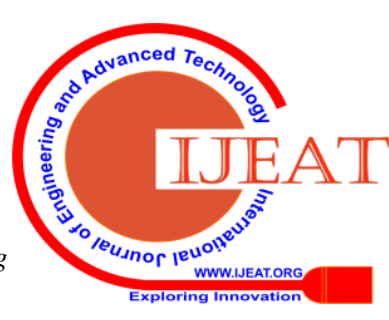


end for

for all $\mathrm{j}, 1 \leq \mathrm{j} \leq \mathrm{N}$ do

compute TCz j based on Equation (10)

end for

estimate Dj based on Equations (11) and (12)

end for

end while

for all $\mathrm{j}, 1 \leq \mathrm{j} \leq \mathrm{N}$ do if $\mathrm{Dj} \geq$ threshold then output ${ }^{\wedge} \mathrm{X} * \mathrm{j}=1$

else

output ${ }^{\wedge} \mathrm{X} * \mathrm{j}=0$

end if

end for

IV. RESULTS AND ANALYSIS

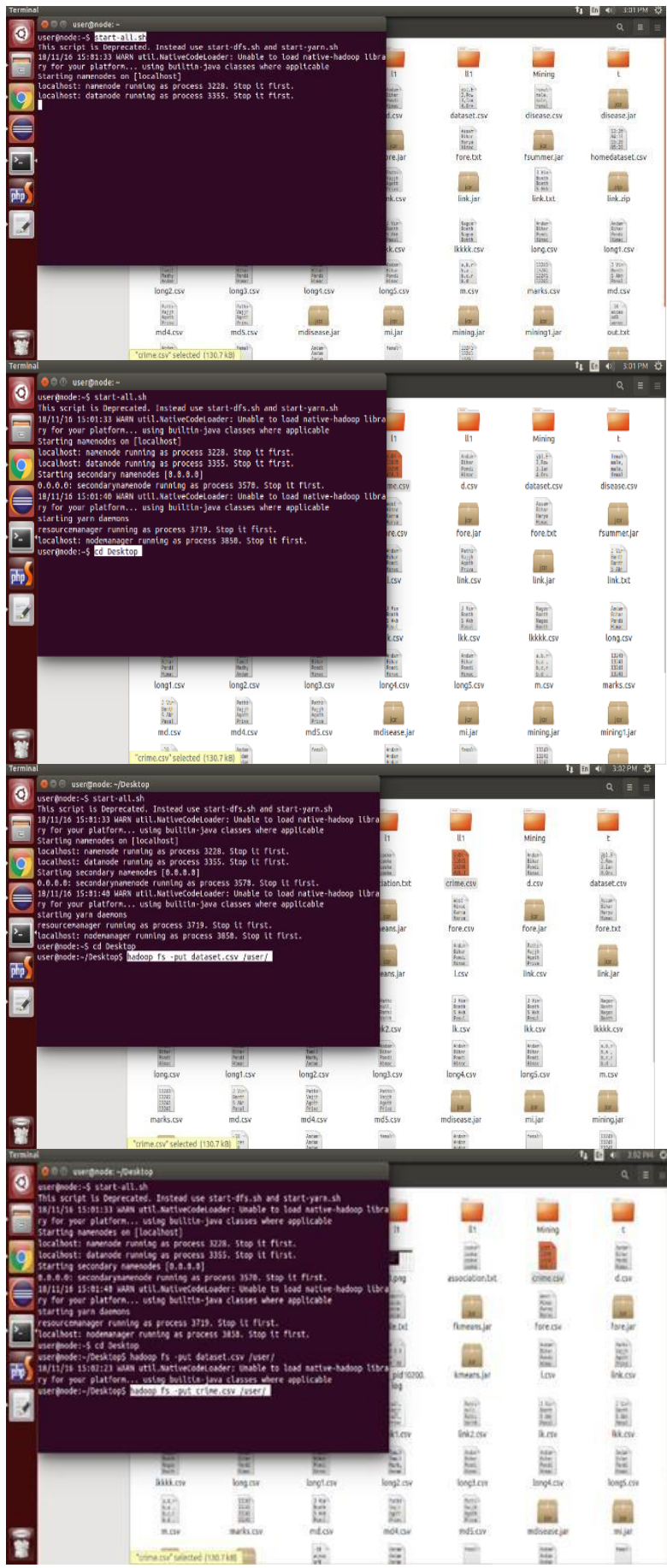

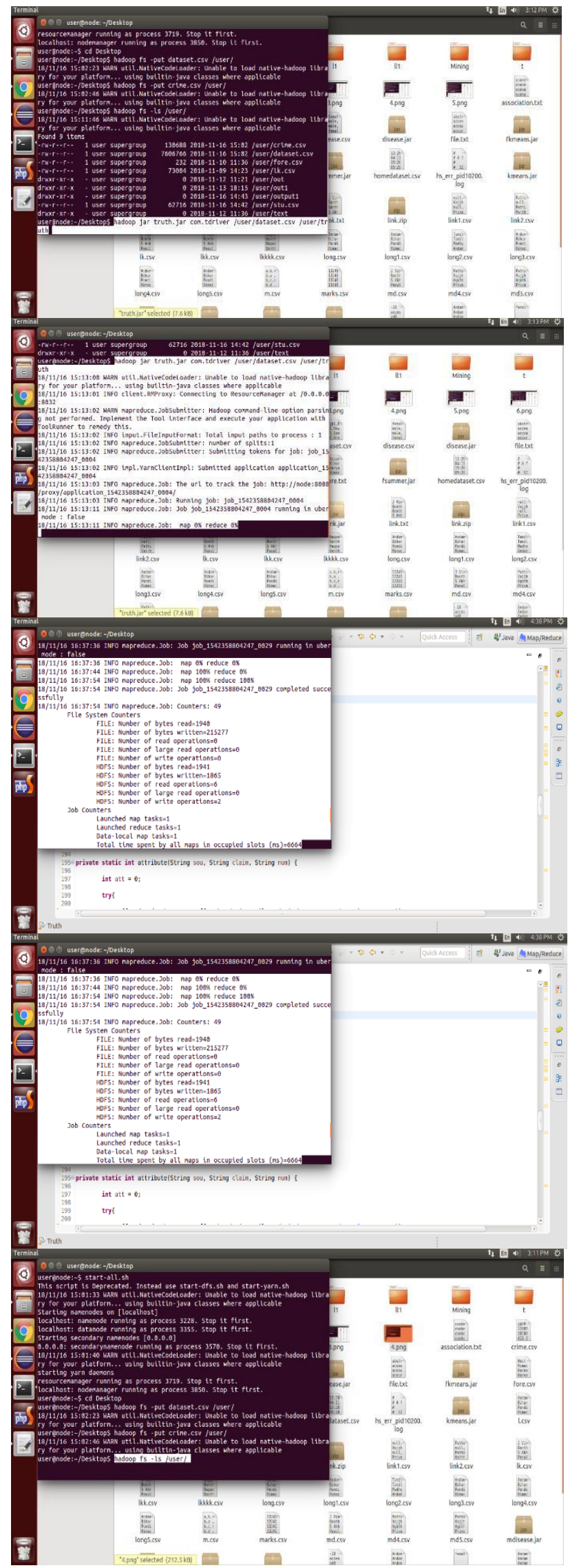

Published By:

DOI: 10.35940/ijeat.B3557.129219

Journal Website: www.ijeat.org

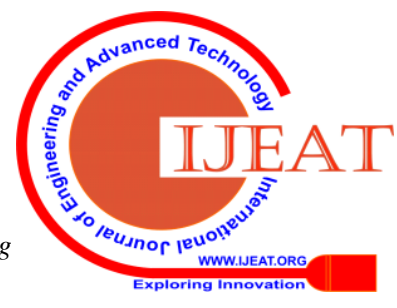




\section{CONCLUSION}

In this paper, we proposed a scalable robust truth discovery (srtd) framework to address the data veracity challenge in big data social media sensing applications. in our solution, we explicitly considered the source reliability, report credibility, and a source's historical behaviors to effectively address the misinformation spread and data sparsity challenges in the truth discovery problem. We also designed and implemented a distributed framework using Work Queue and the HTCondor system to address the scalability challenge of the problem. We evaluated the SRTD scheme using three real- world data traces collected from Twitter. The empirical results showed our solution achieved significant performance gains on both truth discovery accuracy and computational efficiency compared to other stateof-the-art baselines. The results of this paper are important because they provide a scalable and robust approach to solve the truth discovery problem in big data social media sensing applications where data is noisy, unvetted, and sparse.

\section{REFERENCES}

1. P. Bui, D. Rajan, B. Abdul-Wahid, J. Izaguirre, and D. Thain. Work queue+ python: A framework for scalable scientific ensemble applications. In Workshop on python for high performance and scientific computing at sc11, 2011.

2. Z. Z. J. Cheng and W. Ng. Truth discovery in data streams: A single- pass probabilistic approach. In In Proc. of CIKM, pages 1589-1598, 2014.

3. X. L. Dong, L. Berti-Equille, and D. Srivastava. Integrating conflicting data: the role of source dependence. In Proceedings of the VLDB Endowment, pages 550-561, 2009.

4. Q. L. et al. A confidence-aware approach for truth discovery on long- tail data. In Proceedings of the VLDB Endowment, volume 8, pages 425-436, Dec. 2014.

5. Y. L. et al. On the discovery of evolving truth. In Proceedings of the 21th ACM SIGKDD International Conference on Knowledge Discovery and Data Mining - KDD ’15, 2015.

6. R. Farkas, V. Vincze, G.Mora, J. Csirik, and G.Szarvas. The conll2010 shared task: Learning to detect hedges and their scope in natural language text. In In Proceedings of the Fourteenth Conference on Computational Natural Language Learning., 2010.

7. A. Galland, S. Abiteboul, A. Marian, and P. Senellart. Corroborating information from disagreeing views. In In Proc. of the ACM Interna- tional Conference on Web Search and Data Mining (WSDM'10), pages 131-140, 2010.

8. M. Ham, Y. H. Lee, and J. W. Fowler. Integer programming-based real- time scheduler in semiconductor manufacturing. In Winter Simulation Conference, pages 1657-1666. Winter Simulation Conference, 2009.

9. C. Huang and D. Wang. Spatial-temporal aware truth finding in big data social sensing applications. In Proceedings of Trustcom/BigDataSE/ISPA, volume 2, pages 72-79. IEEE, 2015.

10. C. Huang and D. Wang. Topic-aware social sensing with arbitrary source dependency graphs. In International Conference on Information Processing in Sensor Networks (IPSN), pages 1-12. ACM/IEEE, 2016.

11. C. Huang and D. Wang. Critical source selection in social sensing applications. In Distributed Computing in Sensor Systems (DCOSS), 2017 International Conference on to appear. IEEE, 2017.

12. C. Huang, D. Wang, and N. Chawla. Towards time-sensitive truth discovery in social sensing applications. In Proceedings of International Conference on Mobile Ad Hoc and Sensor Systems (MASS), pages 154-

13. 162. IEEE, 2015.

14. C. Huang, D. Wang, and N. Chawla. Scalable uncertainty-aware truth discovery in big data social sensing applications for cyber-physical systems. IEEE Transactions on Big Data, 2017.

15. M. J. Litzkow, M. Livny, and M. W. Mutka. Condor-a hunter of idle workstations. In Distributed Computing Systems, 1988., 8th International Conference on, pages 104-111. IEEE, 1988.

16. J. Marshall, M. Syed, and D. Wang. Hardness-aware truth discovery in social sensing applications. In Distributed Computing in Sensor Systems (DCOSS), 2016 International Conference on, pages 143-152. IEEE, 2016.

17. J. Marshall and D. Wang. Mood-sensitive truth discovery for reliable recommendation systems in social sensing. In Proceedings of Interna- tional Conference on Recommender Systems (Recsys), pages 167-174. ACM, 2016.

18. J. Marshall and D. Wang. Towards emotional-aware truth discovery in social sensing applications. In Smart Computing (SMARTCOMP), 2016 IEEE International Conference on, pages 1-8. IEEE, 2016.

19. nielson. Super bowl 50: Nielsen twitter tv ratings post-game report.

20. R. W. Ouyang, L. M. Kaplan, A. Toniolo, M. Srivastava, and T. Nor- man. Parallel and streaming truth discovery in large-scale quantitative crowdsourcing.

21. J. Qadir, A. Ali, A. Zwitter, A. Sathiaseelan, J. Crowcroft, et al. Crisis analytics: Big data driven crisis response. arXiv preprint arXiv:1602.07813, 2016.

22. L. Rabiner and B. Juang. An introduction to hidden markov models 23. ieee assp magazine, 3(1):4-16, 1986

24. B. T. Rao, N. Sridevi, V. K. Reddy, and L. Reddy. Performance issues of heterogeneous hadoop clusters in cloud computing. arXiv preprint arXiv:1207.0894, 2012

25. D. E. Rivera, M. Morari, and S. Skogestad. Internal model control: Pid controller design. Industrial \& engineering chemistry process design and development, 25(1):252-265, 1986.

26. T. Shelton, A. Poorthuis, M. Graham, and M. Zook. Mapping the data shadows of hurricane sandy: Uncovering the sociospatial dimensions of 'big data'. Geoforum, 52:167-179, 2014.

27. M. Y. S. Uddin, M. T. A. Amin, H. Le, T. Abdelzaher, B. Szymanski, and T. Nguyen. On diversifying source selection in social sensing. In Proc. Ninth Int Networked Sensing Systems (INSS) Conf, pages 1-8, June 2012.

28. A. Viterbi. Error bounds for convolutional codes and an asymptotically optimum decoding algorithm. IEEE Transactions on Information Theory, 13(2):260-269, Apr. 1967.

29. D. Wang, T. Abdelzaher, and L. Kaplan. Surrogate mobile sensing.

30. IEEE Communications Magazine, 52(8):36-41, 2014.

31. D. Wang, T. Abdelzaher, and L. Kaplan. Social sensing: building reliable systems on unreliable data. Morgan Kaufmann, 2015.

32. D. Wang, T. Abdelzaher, L. Kaplan, and C. C. Aggarwal. Recursive fact-finding: A streaming approach to truth estimation in crowdsourcing applications. In The 33rd International Conference on Distributed Computing Systems (ICDCS'13), July 2013.

33. D. Wang, M. T. Al Amin, T. Abdelzaher, D. Roth, C. R. Voss, L. M. Kaplan, S. Tratz, J. Laoudi, and D. Briesch. Provenance-assisted classification in social networks. IEEE Journal of Selected Topics in Signal Processing, 8(4):624-637, 2014.

34. D. Wang, M. T. Amin, S. Li, T. Abdelzaher, L. Kaplan, S. $\mathrm{Gu}$,

35. Pan, H. Liu, C. C. Aggarwal, R. Ganti, X. Wang, P. Mohapatra,

36. B. Szymanski, and H. Le. Using humans as sensors: An estimationtheoretic perspective. In Proc. 13th Int Information Processing in Sensor Networks Symp. IPSN-14, pages 35-46, Apr. 2014.

37. D. Wang and C. Huang. Confidence-aware truth estimation in social sensing applications. In International Conference on Sensing, Commu- nication, and Networking (SECON), pages 336-344. IEEE, 2015.

38. D. Wang, L. Kaplan, and T. F. Abdelzaher. Maximum likelihood analysis of conflicting observations in social sensing. ACM Transactions on Sensor Networks, 10(2):1-27, Jan. 2014.

39. D. Wang, L. Kaplan, H. Le, and T. Abdelzaher. On truth discovery in social sensing: A maximum likelihood estimation approach. In Proc. ACM/IEEE 11th Int Information Processing in Sensor Networks (IPSN) Conf, pages 233-244, Apr. 2012.

40. J. Wang, D. Wang, Y. Zhao, and T. Korhonen. Fast anti-collision algorithms in rfid systems. In Mobile Ubiquitous Computing, Systems, Services and Technologies, 2007. UBICOMM'07. International Confer- ence on, pages 75-80. IEEE, 2007.

41. W. Xue, J. Shi, and B. Yang. X-rime: cloud-based large scale social net- work analysis. In Services Computing (SCC), 2010 IEEE International Conference on, pages 506-513. IEEE, 2010

42. S. R. Yerva, H. Jeung, and K. Aberer. Cloud based social and sensor data fusion. In Information Fusion (FUSION), 2012 15th International Conference on, pages 2494-2501. IEEE, 2012. 
43. X. Yin, J. Han, and P. S. Yu. Truth discovery with multiple conflicting information providers on the web. IEEE Transactions on Knowledge and Data Engineering, 20(6):796-808, June 2008.

44. D. Zhang, H. Rungang, and D. Wang. On robust truth discovery in sparse social media sensing. In Big Data (Big Data), 2016 IEEE International Conference on. IEEE, 2016.

\section{AUTHORS PROFILE}

Mr. Hemadri Atukuru received the B.Tech (CSE) from Sri Venkateswara college of engineering tirupati affiliated to Jawaharlal Nehru Technological University anantapur, India in 2016. He is pursuing his M.Tech in CSE from Chadalavada ramanamma engineering college Tirupati. His areas of interests include Big data, Data structures and Algorithms.

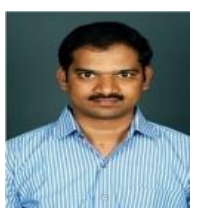

Prof. Janapati Naga Muneiah received the B.Tech (CSE) from Jawaharlal Nehru Technological University, Hyderabad, India in 2001 and M.Tech in CSE from Sri Venkateswara University, Tirupati, India in 2010. He is pursuing his Ph.D in Jawaharlal Nehru Technological University, Kakinada, India in Computer Science and Engineering faculty. He has got 17 years of teaching experience. Presently he is working as Professor and Head of Department of CSE in Chadalawada Ramanamma Engineering college, Tirupati, A.P, India. His areas of interests include Data Mining, Data Warehousing, Big Data, Data Structures and Algorithms. He has guided $11 \mathrm{M}$. Tech theses. He has published more than 10 papers in International journals and some of them are published in SCIE and SCOPUS indexed journals. 